TEKNIK, 42 (1), 2021, 10-19

\title{
Analysis of Leakage Current and Insulator Resistivity for Quality Assurance of Medium Voltage Network Polymer Insulators Alumina - $\mathrm{SiO}_{2}$ in Tropical Climate Simulator Room
}

\author{
Agnes Manik Sari Utami*, Abdul Syakur, Hermawan \\ Departemen Teknik Elektro Fakultas Teknik, Universitas Diponegoro, \\ Jl. Prof. Soedarto, SH, Kampus UNDIP Tembalang, Semarang, Indonesia 50275
}

\begin{abstract}
The tropical climate of Indonesia, which has moderately high rainfall, has an impact on the output of outdoor insulators. The presence of chemical particles, pollen, and salt in the air will bind to the insulating material's outer surface and settle, resulting in crystallization that allows the insulating material's surface roughness to increase. One of the insulators that are being produced is an insulator made of epoxy resin. The injection of fillers is used to further improve the durability of the outdoor epoxy resin insulators installed in tropical climatic conditions. Epoxy resin from bisphenol A-epichlorohydrin and polyaminoamide combined with silane, alumina, and $\mathrm{SiO} 2$ is used as research materials. The parameters examined were leakage current and resistance to insulation. The use of silane as a hardener will also improve the resistivity on the surface of the insulator, which makes it more difficult to flow or reduces the leakage current. Alumina is well-known for being a solid heat and voltage insulator. The addition of SiO2 to the epoxy resin insulating material increases the insulator's mechanical strength in the form of tensile and compressive strength. With fluctuations in temperature and humidity, the artificial tropical environment is replicated in a test chamber. The value of the leakage current increases with an increase in temperature and humidity. The correlation between air temperature and humidity and insulation resistance is inversely proportional, the higher the temperature applied to the insulator, the lower the insulator resistivity. The same refers to the relationship between air humidity and resistance to insulation. The higher the humidity applied to the insulator, the lower the insulator resistivity. At a test voltage of $11 \mathrm{kV}$, a humidity of $60 \%$, and a temperature variation of $25 \mathrm{oC}$, the leakage current of epoxy resin insulators is up to 9.2 uA lower than in a factory-made SiR insulator. The leakage current and insulator resistivity's number is already in the good and protected range such that the insulator can be used and reproduce.
\end{abstract}

Keywords: resin epoxy; leakage currents; insulators resistivity; climate chamber simulator

\section{Introduction}

The use of outdoor polymer insulators would be influenced by temperature and weather. In Indonesia's coastal and agricultural environments, pollutants such as salt, dust, and chemicals will bind to the surface of the insulator, causing crystallization resulting in increased surface roughness of the insulating layer. Contamination of the insulator surface is a significant concern for the efficiency of the outdoor insulator. In ambient environments with a high degree of emissions and humidity, the pollutant coating attached to the soil will become wet. This causes leakage currents to flow,

\footnotetext{
*) Author Correspondence:

E-mail: agneeesmanik@gmail.com
}

which causes the substrate to heat the pollutant (Badachi et al, 2016). Due to the persistent leakage current, the contaminant coating connected to the insulator surface will form a dry strip. This state will allow the discharge of the charge to leak into the dry band at certain voltages. The discharge arc may be extended to allow the flashover to occur over the entire surface (Xinhan et al, 2020). Ceramics (porcelain), glass, and polymers, with some dissension and benefits, are the most popular and commonly used insulation materials in Indonesia (Syakur et al, 2017). The benefits of ceramic and glass insulating materials are high thermal ability and low thermal conductivity, corrosion-resistant, hard and solid, but ceramic and glass insulating materials 


\section{TEKNIK, 42 (1), 2021, 11}

have a physical weakness that is heavy and waterabsorbing (hygroscopic) such that the leakage of current on the surface will easily cause a flashover. Polymer has the ability to replace the use of ceramics as an insulating material (Gao et al, 2019). Research on the electrical parameters of the insulators, namely the leakage current, is required to determine the longterm capability of the outdoor epoxy resin insulators. The key factor influencing the efficiency of polymer insulating materials is their hydrophobic effect. Although similar to ceramic or glass materials, polymer insulation materials have the benefit of dilution, tolerance to volume and strong thermal properties, comparatively light construction, high acid resistance, and hydrophobic properties, due to lack of weatherproof resistance, they can result in reduced mechanical strength as well as being susceptible to contaminants (Gao et al, 2019). This research examined the efficiency of epoxy resin test insulators with alumina filler and $\mathrm{SiO} 2$ (silica sand). The research materials used in this analysis were epoxy resins made from polyaminoamide (PAMAM) and DGEBA (bisphenol A - epichlorohydrin) and then mixed with silane, alumina, and $\mathrm{SiO} 2$. The kind of insulator used is an insulator with a uniform shed and a number of fins up to three parts. Research on the electrical properties of epoxy resin polymer insulators with silicone oxide and alumina fillers, in particular in conditions of temperature and humidity variance, has not been completely and thoroughly performed. Characteristics of the type of suspension insulator can be tested by providing for different temperatures and artificial humidity by comparison to the IEC 61109 specification and testing that has been and is currently being carried out both at home and abroad.

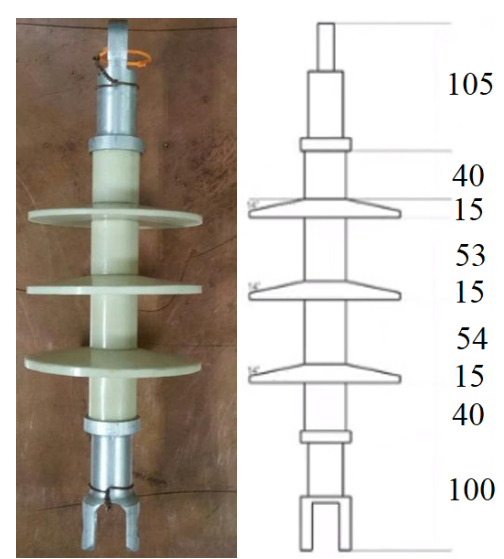

Figure 1. Insulator test (measurement in $\mathrm{mm}$ )

\section{Materials and Methods}

The sample used in this analysis was a $20 \mathrm{kV}$ (uniform shed) epoxy resin insulator. In this study, the suspension insulator is attached to a $20 \mathrm{kV}$ mediumvoltage network. Measurements are performed using a frequency of $50 \mathrm{~Hz}$ and high voltage to achieve leakage currents on the surface of the test insulator. The use of high voltage ensures that there is ample electrical pressure (electric field strength) on the surface of the insulator. For epoxy resin insulators using the simple polymer DGEBA (Diglycidyl Ether Bisphenol-A) epoxy resin polymer, MPDA (Metaphenylene-diamine) or hardening agent, silicon oxide, and alumina. The addition of silica to the insulating material of the epoxy resin helps to increase the mechanical strength, respectively the tensile strength and the compressive strength. Alumina is a fine electrical insulator, although its thermal conductivity is comparatively high $(40 \mathrm{~W} / \mathrm{mK})$. The test insulator used is shown in Figure 1.

In the test, the insulator is put in a glass chamber test $(80 \times 80 \times 100) \mathrm{cm}$ with $5 \mathrm{~mm}$ thickness. The test chamber is fitted with a temperature and humidity control system, a mist system, and a heating system. For the same research treatment, i.e. dry and wet weather monitoring. The chamber test used is shown in Figure 2.

The leakage current model of the insulator illustrates linear conditions where the leakage current is a pure sine wave. In transmission line insulators, the resistive component has a more dominant effect than the capacitive component. This is because the level of usage of the insulator is $50 \mathrm{~Hz}$, so the skin effect and the power are low and steady. However, the leakage current is not necessarily linear, resulting in a leakage current in the shape of a pure sine wave. In certain

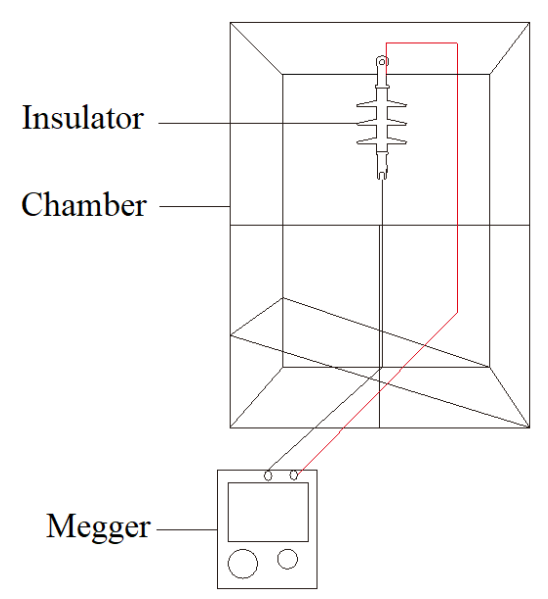

Figure 2. Chamber test 


\section{TEKNIK, 42 (1), 2021, 12}

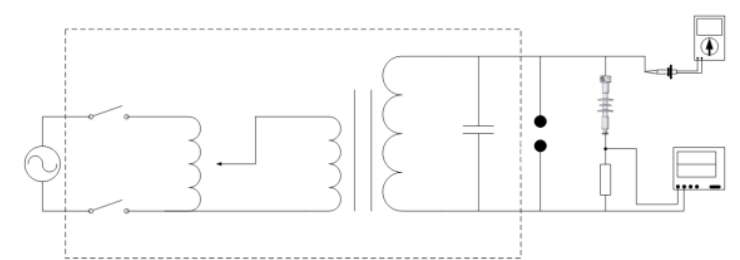

Figure 3. The leakage current test circuit (Zhang et al, 2018)

situations, such as where small dry-band arcs tend to emerge on the insulator surface, the leakage current waveform is not linear because it is distorted by the pure sine wave in its original form. When the corona occurs on the top of the insulator, a surge appears in the present wave of leakage.

The existing leakage current standard for insulators is not currently defined by any standardization organization. The key explanation is that the insulator is often mounted at a height that does not interfere with human activities, so the standards for the leakage current of the insulator are based more on the efficiency of the operation of the power system than on protection. In such a way that the cap on the amount of the leakage current is generally fixed by the State electricity company concerned. Of course, the cap for the value of the existing leakage ranges from country to country. Limits depend on the type of insulator used, the rated voltage, and the permitted line losses. Generally, the highest permitted spike leakage current is $100 \mathrm{~mA}$, while observed values vary from a few micrometers to $50 \mathrm{~mA}$ in dry conditions, wave leakage can be up to $50 \mathrm{~mA}$ or more in rainy weather conditions. Those are still considered safe (Taryo et al, 2018).

Leakage current is current that flows in the protective earth conductor or chassis. When there is no ground connection or it is broken, this leakage current can flow through the human body, and thus it is

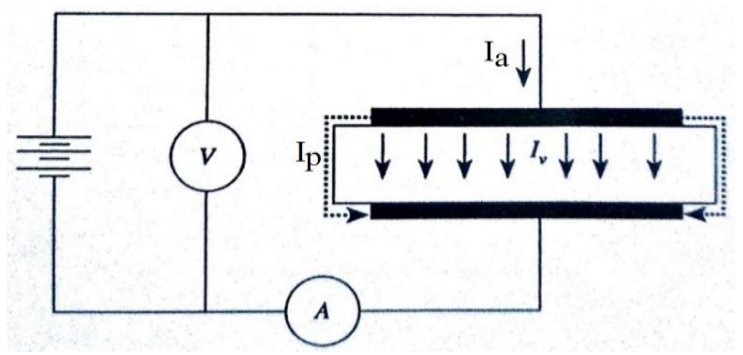

Figure 5. Current in a solid insulating material

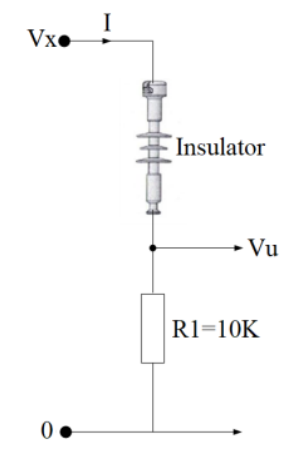

Figure 4. Voltage divider circuit (Arismunandar, 2009)

important to limit. When it comes to leakage current there's not only a difference in the allowed current but also in how the devices are classified. IEC 60335-1 identifies two categories; portable appliances and stationary appliances. Portable appliances have the same requirements as hand-held devices $(0.75 \mathrm{~mA})$, while stationary and movable devices of IEC 60950-1 have the same requirements as the stationary appliances of IEC 60335-1 (3.5 mA). To obtain data on the characteristics of the leakage current from each sample, the leakage current test was carried out using the test circuit as shown in Figure 3.

Observation of leakage currents includes the use of an oscilloscope as equipment. The voltage that reaches the oscilloscope must be compatible with the characteristics of the oscilloscope. The voltage divider circuit is mounted as an oscilloscope safety mechanism used to reduce the voltage that reaches the oscilloscope. The voltage divider circuit used is shown in Figure 4.

There are two resistors in the voltage divider circuit, namely the insulator as a high voltage resistor $(\mathrm{Rh})$ and the $10 \mathrm{~K} \Omega$ as a low voltage resistor as a $10 \mathrm{~W}$ rock resistor ( $\mathrm{Rl})$. $\mathrm{Vu}$ is the output voltage read on the oscilloscope, and $\mathrm{I}$ is the leakage current in the insulator. The value of the leakage current can be found using Equation 1.

$$
I=\frac{V u}{10 K}
$$

The test protocol for leakage current was planned as follows: before joining the test, the test substance was washed and cleaned with purified water and a clean cloth. The insulator has to be clean from dust. Set the test chamber air temperature and humidity values. For the voltage variable, the temperature and humidity for the test are conditioned at $25^{\circ} \mathrm{C}$ and the air humidity is $60 \%$. If the conditions are right, install the insulator in the testing chamber 


\section{TEKNIK, 42 (1), 2021, 13}

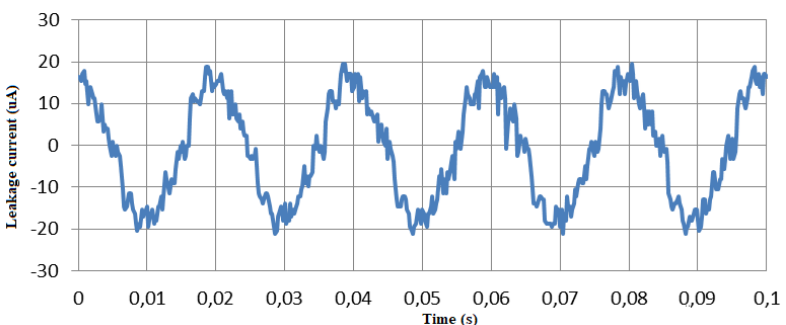

(a) Test voltage $11 \mathrm{kV}$

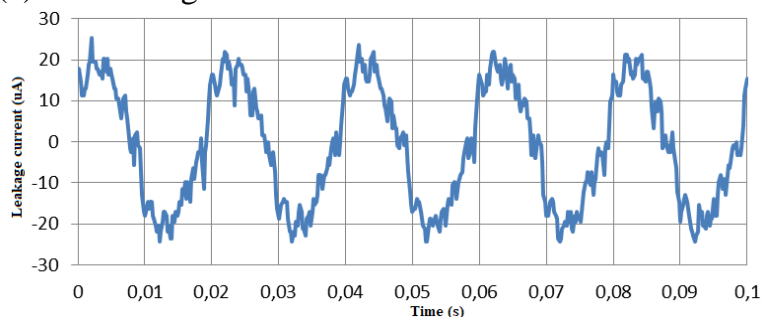

(c) Test voltage $13 \mathrm{kV}$

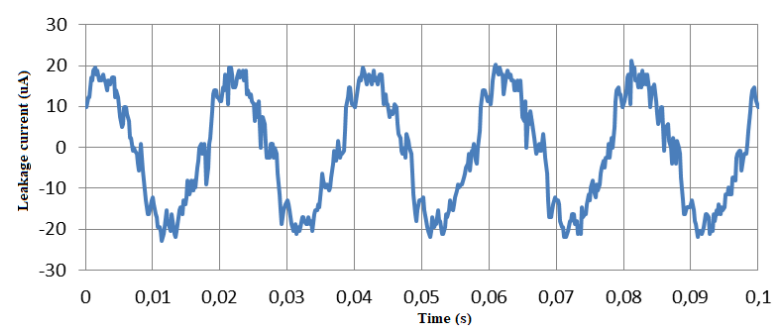

(b) Test voltage $12 \mathrm{kV}$

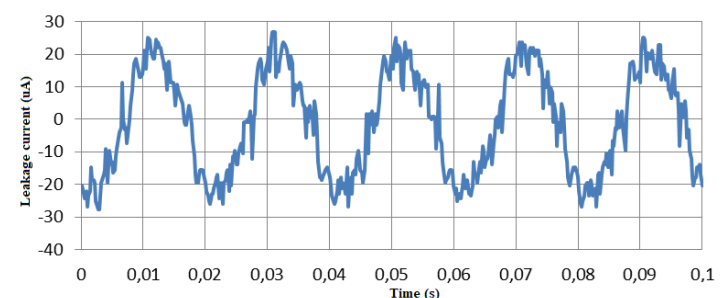

(d) Test voltage $14 \mathrm{kV}$

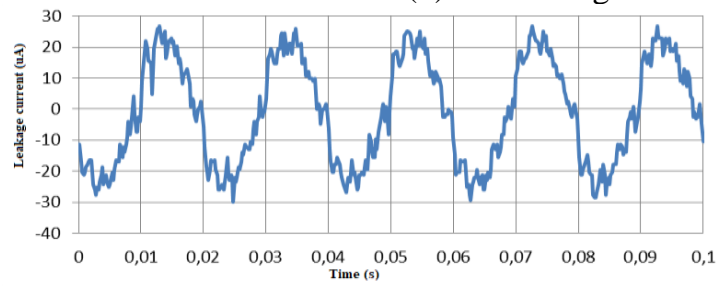

(e) Test voltage $15 \mathrm{kV}$

Figure 6. Graph of the leakage current of the insulator with various test voltages (a) $11 \mathrm{kV}$, (b) $12 \mathrm{kV}$, (c) $13 \mathrm{kV}$, (d) $14 \mathrm{kV}$, and (e) $15 \mathrm{kV}$

and prepare other testing equipment. Apply the test voltage in stages, from $11 \mathrm{kV}, 12 \mathrm{kV}, 13 \mathrm{kV}, 14 \mathrm{kV}$ until $15 \mathrm{kV}$. The test voltage used in stages should be $11.547 \mathrm{kV}-10 \%, 11.547 \mathrm{kV}$, and $11.547 \mathrm{kV}+5 \%$, where this value is the phase-neutral voltage (line voltage) of the $20 \mathrm{kV}$ medium voltage network, namely $11.547 \mathrm{kV}$ with a minimum of $-10 \%$ and a maximum. $+5 \%$. For each voltage variation, save the leakage current waveform data into the laptop to be processed using Microsoft Office Excel 2013 and repeat 3 times. For the temperature variable, the data were taken by changing the temperature variable in the test chamber to $25^{\circ} \mathrm{C}, 28^{\circ} \mathrm{C}, 31^{\circ} \mathrm{C}, 34^{\circ} \mathrm{C}$, and $37^{\circ} \mathrm{C}$ and by maintaining the humidity at $60 \%$. For the humidity variable, the data were taken by changing the humidity component in the test chamber to $60 \%, 70 \%, 80 \%$, $90 \%$, and $100 \%$ and keeping the temperature at $25^{\circ} \mathrm{C}$.

If the insulating material is given a direct voltage as seen in Figure 5, the current flowing in the insulating material consists of two elements, those are the current flowing on the surface of the insulating material called the surface current (Ip) and the current flowing through the volume of the insulating material is called the flow of volume (Iv). Thus, the source current can be written as in Equation 2.

$$
I_{\mathrm{a}}=I_{\mathrm{V}}+I_{\mathrm{p}}
$$

The resistance experienced by volume currents is referred to as volume resistance (Rv), while the resistance experienced by surface currents is referred to as surface resistance (Rp). The properties of the insulating material that determine the value of the two resistances are volume resistance and surface resistance, respectively.

Megger (Mega ohm Meter) is the unit used for the insulation resistance test. Megger tests resistance to insulation in Mega Ohms (M $\Omega)$. It is advisable to use the same voltage as the operating voltage when calculating insulation resistance, but the conditions in the field are different. Due to the shortcomings of the measuring instruments, $5 \mathrm{kV}$ measuring instruments are used for measuring insulation resistance with a voltage of $20 \mathrm{kV}$. Equation 3 can be used to determine the minimum standard value of the insulation resistance measurement (Tobing, 2012).

$$
R=\frac{1000 \cdot\left(v^{2}\right)}{E} \cdot \frac{2,5}{\left(10^{6}\right)} \quad(\mathrm{M} \Omega)
$$




\section{TEKNIK, 42 (1), 2021, 14}

Table 1. Measurement of voltage variation leakage current

\begin{tabular}{ccccc}
\hline $\begin{array}{c}\text { Test } \\
\text { Voltages } \\
(\mathbf{k V})\end{array}$ & $\begin{array}{c}\text { Data } \\
\mathbf{1}\end{array}$ & Data 2 & Data 3 & Average \\
\hline 11 & 12.30 & 12.17 & 12.21 & 12.23 \\
12 & 13.40 & 13.26 & 13.30 & 13.32 \\
13 & 14.38 & 14.38 & 14.49 & 14.31 \\
14 & 15.83 & 15.88 & 16.11 & 15.94 \\
15 & 16.88 & 16.74 & 16.78 & 16.80 \\
\hline
\end{tabular}

The minimum insulation resistance $(\mathrm{M} \Omega)$ can be obtained from squaring the $\mathrm{v}$ as the working voltage, then multiplied by 1000 as a multiplier factor and 2.5 as a safety factor. The last step is it should be divided by $\mathrm{E}$ as the value of the Megger voltage (Volt).

\section{Results and Discussions}

This chapter presents results and discussion of data collected from the measurement and test that is analyzed. The research was carried out to assess the performance of electrical insulators in terms of temperature and humidity.

\subsection{Leakage Current}

When an insulating material is exposed to an electric field, current flows on the insulating material's surface. This current is often referred to as the leakage current or creepage current. The surface resistance of the insulating material determines the amount of leakage current (Suwarno, 2016).

\subsubsection{Leakage current with variable voltage changes}

There are five voltage variants used, namely 11 $\mathrm{kV}, 12 \mathrm{kV}, 13 \mathrm{kV}, 14 \mathrm{kV}$, and $15 \mathrm{kV}$. Measurements were made by setting the test chamber at an average Indonesian temperature of $25^{\circ} \mathrm{C}$ with a humidity of $60 \%$.

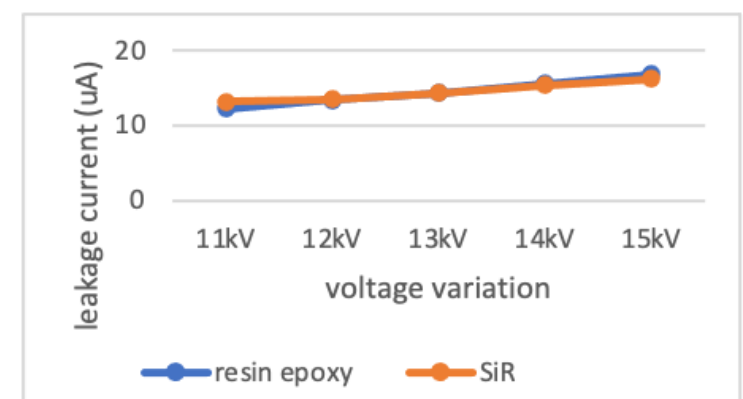

Figure 7. Data average leakage current epoxy resin insulator and $\mathrm{SiR}$ insulator in variable voltage
Table 2. Measurement of voltage variation leakage current SiR insulator (Utami. 2018)

\begin{tabular}{ccccc}
\hline $\begin{array}{c}\text { Test } \\
\text { Voltages } \\
(\mathbf{k V})\end{array}$ & \multicolumn{4}{c}{ Leakage Current (uA) } \\
\hline 11 & 13.214 & 13.207 & 13.143 & 13.188 \\
12 & 13.507 & 13.308 & 13.417 & 13.411 \\
13 & 14.356 & 14.464 & 14.192 & 14.337 \\
14 & 15.124 & 15.508 & 15.409 & 15.347 \\
15 & 16.493 & 16.029 & 15.928 & 16.150 \\
\hline
\end{tabular}

The product of the leakage current value for voltage variations is shown in Figure 6.

Figure 6 presents one of the outcomes of testing the leakage current wave against voltage variations of 11 $\mathrm{kV}, 12 \mathrm{kV}, 13 \mathrm{kV}, 14 \mathrm{kV}$, and $15 \mathrm{kV}$ at temperatures of $25^{\circ}$ degrees Celsius and a humidity of $60 \%$ on an epoxy resin insulator. For each voltage difference, measurements were taken three times and the average was displayed in Table 1 . As a comparison, a factorymade SiR insulator is used. Table 2 displays the results and the data of the leakage current test.

A graph can be generated using the data in Tables 1 and 2, as seen in Figure 7. This graph compares the value of the leakage current that occurs on the epoxy resin insulator and $\mathrm{SiR}$ insulator to the value of the applied test voltage.

Figure 7 indicates that as the test voltage applied to the test insulator is increased, the value of the leakage current is also increased. The explanation is that as the voltage applied to the insulating material increases, the discharge on the surface of the insulator will also rise. The relationship between voltage and leakage current is directly proportional, the higher the voltage applied to the insulator, the higher the leakage current generated.

\subsubsection{Leakage current with changes in temperature variables}

Retrieval of leakage current data with changes in the environment variable temperature around the insulator. The data were taken by changing the temperature variable in the test chamber to $25^{\circ} \mathrm{C}, 28^{\circ} \mathrm{C}$, $31^{\circ} \mathrm{C}, 34^{\circ} \mathrm{C}$, and $37^{\circ} \mathrm{C}$ and by maintaining the humidity at $60 \%$. For the calculation of leakage current. this temperature difference applies a test voltage of 11.547 $\mathrm{kV}$ since it is a phase-neutral voltage of a $20 \mathrm{kV}$ medium-voltage network. i.e. $11.547 \mathrm{kV}$ with a minimum tolerance of $-10 \%$ and a maximum voltage of $+5 \%$ based on IEC 60038 Standard Voltage. The product of the leakage current value for temperature variations is shown in Figure 8. 


\section{TEKNIK, 42 (1), 2021, 15}

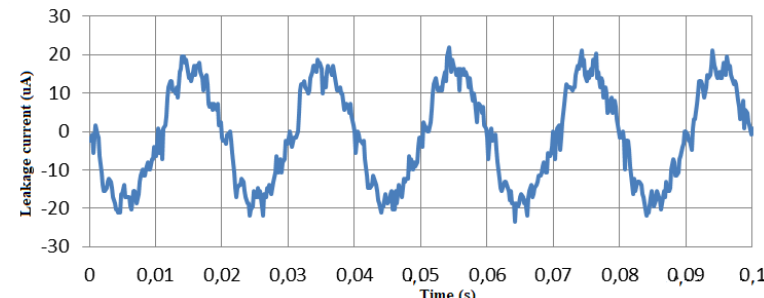

(a) Test temperature $25^{\circ} \mathrm{C}$

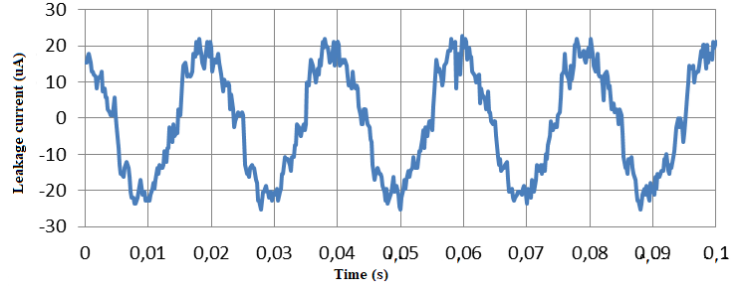

(c) Test temperature $31^{\circ} \mathrm{C}$

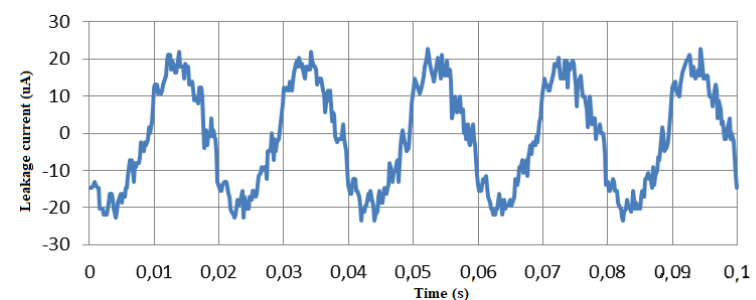

(b) Test temperature $28^{0} \mathrm{C}$

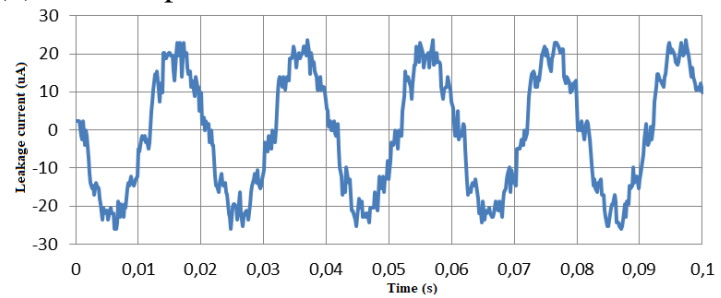

(d) Test temperature $34^{0} \mathrm{C}$

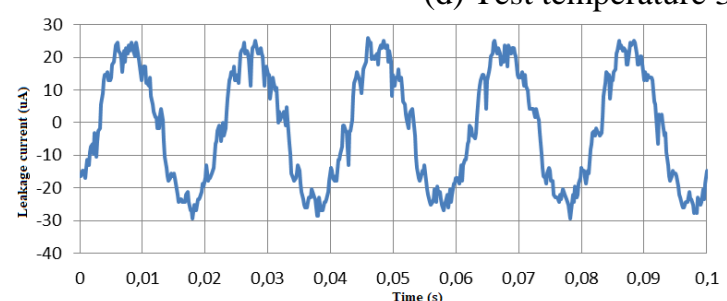

(e) Test temperature $37^{0} \mathrm{C}$

Figure 8. Graph of insulator leakage current with test voltage $11.547 \mathrm{kV}$ temperature variation (a) $25^{\circ} \mathrm{C}$, (b) $28^{0} \mathrm{C}$, (c) $31^{\circ} \mathrm{C}$, (d) $34^{\circ} \mathrm{C}$, and (e) $37^{\circ} \mathrm{C}$

Figure 8 presents one of the outcomes of testing the leakage current wave against temperature variations of $25^{\circ} \mathrm{C}, 28^{\circ} \mathrm{C}, 31^{\circ} \mathrm{C}, 34^{\circ} \mathrm{C}$, and $37^{\circ} \mathrm{C}$ at voltages of $11 \mathrm{kV}$ and a humidity of $60 \%$. For each voltage difference, measurements were taken three times and the average was displayed in Table 3.

As a comparison, Table 4 display the data of the leakage current of factory-made SiR insulators were tested with variations temperature $20^{\circ} \mathrm{C}, 25^{\circ} \mathrm{C}$, $30^{\circ} \mathrm{C}, 35^{\circ} \mathrm{C}$, and $40^{\circ} \mathrm{C}$ on a test voltage of $11 \mathrm{kV}$ and humidity $60 \%$. There are several different temperatures used, although it doesn't matter because they are all in the same range and are balanced.

Based on the data in Table 3 and Table 4, a

Table 3. Measurement of temperature variation leakage current

\begin{tabular}{ccccc}
\hline Temp & & \multicolumn{4}{c}{ Leakage Current (uA) } \\
('C) & Data 1 & Data 2 & Data 3 & Average \\
\hline 25 & 12.70 & 12.80 & 12.67 & 12.73 \\
27 & 13.98 & 13.87 & 13.88 & 13.91 \\
31 & 14.74 & 14.25 & 14.08 & 14.36 \\
34 & 15.57 & 15.59 & 15.23 & 15.46 \\
37 & 17.46 & 17.48 & 17.25 & 17.40 \\
\hline
\end{tabular}

graph can be generated as seen in Figure 9. This graph compares the value of the leakage current that occurs on the epoxy resin insulator and $\mathrm{SiR}$ insulator to the value of the applied temperature.

Figure 9 indicates that as the temperature of the test chamber added to the test insulator is increased, the value of the leakage current is also increased. The explanation for this is that as the temperature applied to the insulating material increases resulting in the release of free electrons trapped in the insulating material, these electrons become current carriers. The insulator has a negative temperature coefficient such that the resistance to insulation decreases as temperature rises and the

Table 4. Measurement of voltage variation leakage current SiR insulator (Utami. 2018)

\begin{tabular}{ccccc}
\hline Temp & & \multicolumn{3}{c}{ Leakage Current (uA) } \\
$\left({ }^{\mathbf{0}} \mathbf{C}\right)$ & Data 1 & Data 2 & Data 3 & Aver \\
\hline 20 & 19.08 & 19.83 & 20.29 & 19. \\
25 & 21.815 & 21.67 & 22.29 & 21. \\
30 & 22.67 & 22.39 & 23.23 & 22. \\
35 & 23.48 & 23.96 & 23.82 & 23. \\
40 & 24.94 & 24.77 & 24.83 & 24. \\
\hline
\end{tabular}




\section{TEKNIK, 42 (1), 2021, 16}

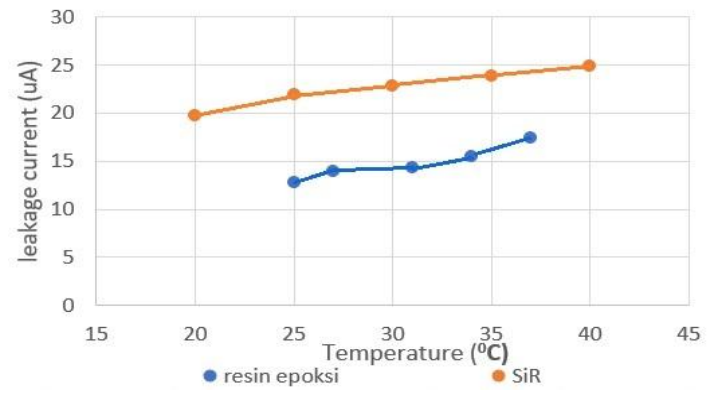

Figure 9. Data average leakage current resin epoxy insulator and $\mathrm{SiR}$ insulator in the temperature variable

leakage current increases. It can be inferred that the relationship between temperature and leakage current is proportionate and the higher the temperature applied to the insulator, the higher the current of leakage.

\subsubsection{Leakage current with variable change in air humidity}

Retrieval of leakage current data with changes in air humidity around the insulator. The data were taken by changing the humidity component in the test

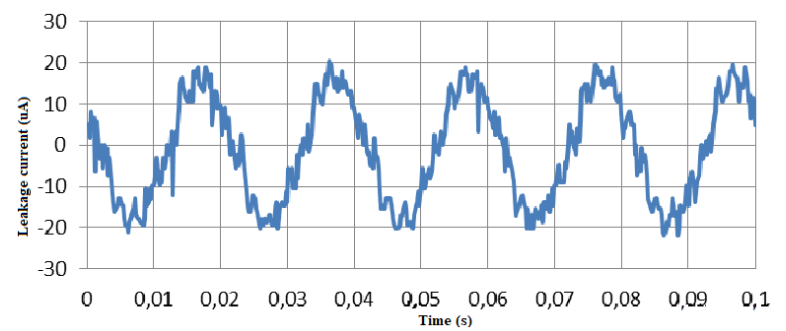

(a) Humidity $60 \%$

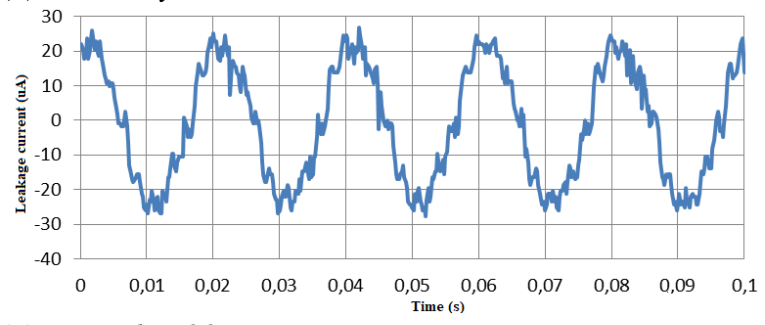

(c) Humidity $80 \%$
Table 5. Measurement of the leakage current of humidity variations

\begin{tabular}{ccccc}
\hline Humidity & & \multicolumn{3}{c}{ Leakage current (uA) } \\
$(\boldsymbol{\%})$ & Data 1 & Data 2 & Data 3 & Average \\
\hline 60 & 11.21 & 11.34 & 11.25 & 11.27 \\
70 & 13.02 & 12.73 & 12.79 & 12.84 \\
80 & 16.26 & 15.42 & 15.41 & 15.70 \\
90 & 29.34 & 30.41 & 28.16 & 29.31 \\
100 & 80.42 & 69.14 & 64.95 & 71.51 \\
\hline
\end{tabular}

chamber to $60 \%, 70 \%, 80 \%, 90 \%$, and $100 \%$ and keeping the temperature at $25^{\circ} \mathrm{C}$. In the calculation of the leakage current. this humidity difference applies a test voltage of $11.547 \mathrm{kV}$ since it is a phase-neutral voltage of a $20 \mathrm{kV}$ medium voltage network of 11.547 $\mathrm{kV}$ with a minimum tolerance of $-10 \%$ and a limit of $+5 \%$ based on IEC 60038 Standard Voltage. The product of the leakage current value for humidity variations is shown in Figure 10.

Figure 10 presents one of the outcomes of

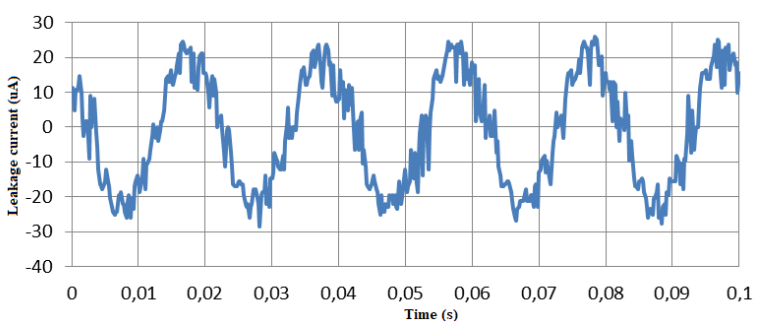

(b) Humidity $70 \%$

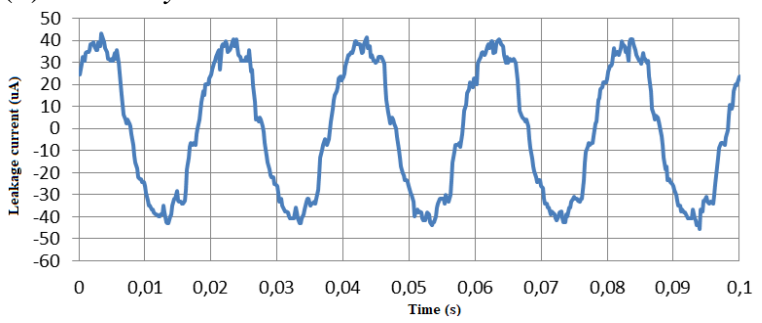

(d) Humidity $90 \%$

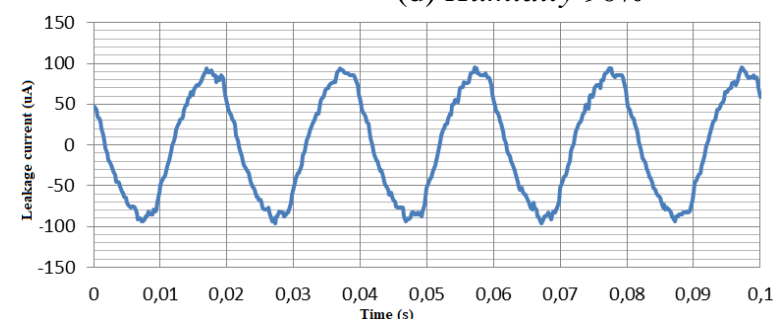

(e) Humidity $100 \%$

Figure 10. Graph of the leakage current of the insulator with a test voltage of $11.547 \mathrm{kV}$ humidity variations (a) $60 \%$, (b) $70 \%$, (c) $80 \%$, (d) $90 \%$, (e) $100 \%$ 


\section{TEKNIK, 42 (1), 2021, 17}

Table 6. Measurement of the leakage current of humidity variations(Utami. 2018)

\begin{tabular}{ccccc}
\hline Humidity & \multicolumn{4}{c}{ Leakage current (uA) } \\
$(\boldsymbol{\%})$ & Data 1 & Data 2 & Data 3 & Average \\
\hline 50 & 18.74 & 18.42 & 19.13 & 18.77 \\
60 & 19.01 & 19.37 & 20.44 & 19.61 \\
70 & 21.59 & 20.69 & 22.57 & 21.62 \\
80 & 21.79 & 22.65 & 23.92 & 22.79 \\
90 & 23.58 & 24.57 & 25.65 & 24.60 \\
\hline
\end{tabular}

testing the leakage current wave against humidity variations $60 \%, 70 \%, 80 \%, 90 \%, 100 \%$ at the voltage of $11 \mathrm{kV}$ and a humidity of $60 \%$. For each voltage difference, measurements were taken three times and the average was displayed in Table 5.

As a comparison, data displayed in Table 6 contains the leakage current of factory-made $\mathrm{SiR}$ insulators were tested with variations humidity $50 \%$, $60 \%, 70 \%, 80 \%$, and $90 \%$ on a test voltage of $11 \mathrm{kV}$ and temperature $25^{\circ} \mathrm{C}$. There are several different humidities used, although it doesn't matter because they are all in the same range and are balanced

Based on the data in Table 5 and Table 6, a graph can be made and shown in Figure 11. This graph compares the value of the leakage current that occurs on the epoxy resin insulator and SiR insulator to the value of the applied humidity.

Figure 11 indicates that as the humidity of the test chamber added to the test insulator is increased, the value of the leakage current is also increased. The explanation is that as the added air humidity increases, more water vapor is captured by the insulator surface which increases the surface conductivity of the insulator so that the current can pass more easily. It can be inferred that the relationship between air humidity and leakage present is directly

Table 7. Measurement of insulator resistivity to temperature fluctuations

\begin{tabular}{ccccc}
\hline $\begin{array}{c}\text { Temp } \\
\left({ }^{\mathbf{0}} \mathbf{C}\right)\end{array}$ & Data 1 & Data 2 & Data 3 & Average \\
\hline 25 & 34.2 & 27.3 & 24.5 & 28.6 \\
27 & 29.7 & 27.5 & 21.1 & 26.1 \\
31 & 23 & 21.1 & 21.2 & 21.77 \\
34 & 18.7 & 17.9 & 19.2 & 18.6 \\
37 & 14.6 & 14.8 & 16.4 & 15.27 \\
\hline
\end{tabular}

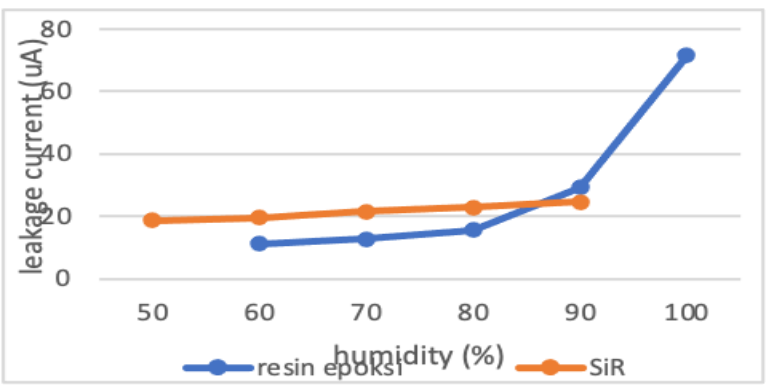

Figure 11. Data average leakage current resin epoxy insulator and $\mathrm{SiR}$ insulator in humidity variable

proportional to the greater the humidity of the air added to it. Insulator. the current of leakage will increase. Especially at $100 \%$ humidity, the water vapor in the test chamber is too saturated, resulting in a sharp increase in the value of the leakage current.

\subsection{Insulator Resistivity}

The Megger is the equipment used in the insulation resistance test (Mega ohm meter). Mega $\mathrm{Ohm}(\mathrm{M} \Omega)$ units are used by Megger to measure insulation resistance (Tobing, 2012).

\subsubsection{Insulator resistivity with variable change in temperature}

Retrieval of insulation resistance data with improvements in temperature variables by changing the temperature variables in the test chamber to $25^{\circ} \mathrm{C}$, $28^{\circ} \mathrm{C}, 31^{\circ} \mathrm{C}, 34^{\circ} \mathrm{C}$, and $37^{\circ} \mathrm{C}$ and maintaining the humidity at $60 \%$. A test voltage of $5 \mathrm{kV}$ is used to calculate the insulation tolerance of this temperature variation. For each temperature difference, measurements were taken three times and the average was displayed in Table 7.

A graph can be drawn from the data in Table 7 in Figure 12. This graph presents outcomes of testing the insulator resistivity against temperature variations $25^{\circ} \mathrm{C}, 27^{\circ} \mathrm{C}, 31^{\circ} \mathrm{C}, 34^{\circ} \mathrm{C}$, and $37^{\circ} \mathrm{C}$ on a test voltage of

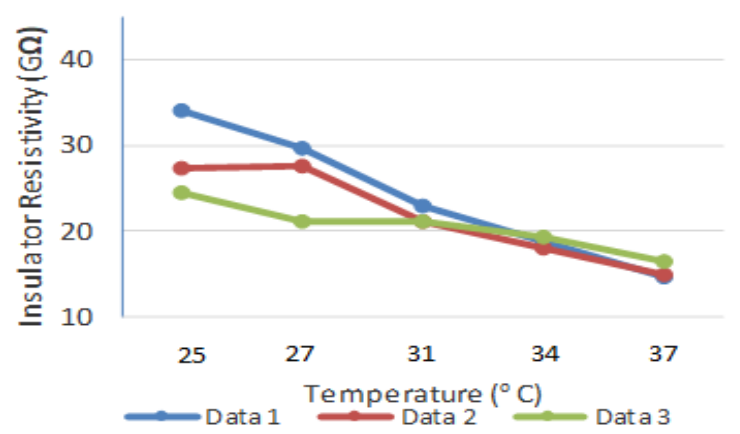

Figure 12. Data from the calculation of insulation resistivity of temperature differences in uniform fine insulators 


\section{TEKNIK, 42 (1), 2021, 18}

Table 8. Measurement of humidity variation insulation resistance

\begin{tabular}{ccccc}
\hline \multirow{2}{*}{ Humidity } & \multicolumn{4}{c}{ Insulator resistivity (G $\mathbf{\Omega})$} \\
& Data & Data & Data & Average \\
& $\mathbf{1}$ & $\mathbf{2}$ & $\mathbf{3}$ & \\
\hline 60 & 30.1 & 29.7 & 29.3 & 29.7 \\
70 & 5.95 & 5.60 & 5.46 & 5.67 \\
80 & 1.67 & 1.54 & 1.51 & 1.57 \\
90 & 0.53 & 0.52 & 0.49 & 0.51 \\
100 & 0.27 & 0.24 & 0.20 & 0.23 \\
\hline
\end{tabular}

$5 \mathrm{kV}$ and humidity $60 \%$ on an epoxy resin insulator.

From the insulation resistance data seen in Table 7 and Figure 12, it can be shown that the insulation resistance value decreases as the temperature difference added to the insulator is increased. This is because as the temperature applied to the insulating layer increases, resulting in the release of free electrons in the insulating material, these electrons become current carriers. In Khircoff law, the value of the current is inversely proportional to the value of the resistance. It can also be inferred that the relationship between temperature and insulation resistance is inversely proportional, the higher the temperature applied to the insulator, the lower the resistance.

\subsubsection{Isolation resistivity with changes in humidity variables}

Measurement of insulation resistance with humidity variations represents the changing conditions of environmental humidity around the insulator. Measurements were made by varying the humidity in the test chamber from $60 \%, 70 \%, 80 \%$, $90 \%, 100 \%$ and keeping the temperature at $25^{\circ} \mathrm{C}$. The test voltage applied is $5 \mathrm{kV}$. For each humidity difference, measurements were taken three times and the average was displayed in Table 8 .

The graph in Figure 13 can be rendered by using the details from Table 8 . This graph presents outcomes of testing the insulator resistivity against humidity variations, $60 \%, 70 \%, 80 \%, 90 \%$, and $100 \%$ on a test voltage of $5 \mathrm{kV}$ and temperature $25^{\circ} \mathrm{C}$ on an epoxy resin insulator.

From the insulation resistance data seen in Table 8 , it can be shown that the resistance value decreases as the humidity variance added to the test insulator are increased. It can be shown that the insulation resistance value continues to decrease as the

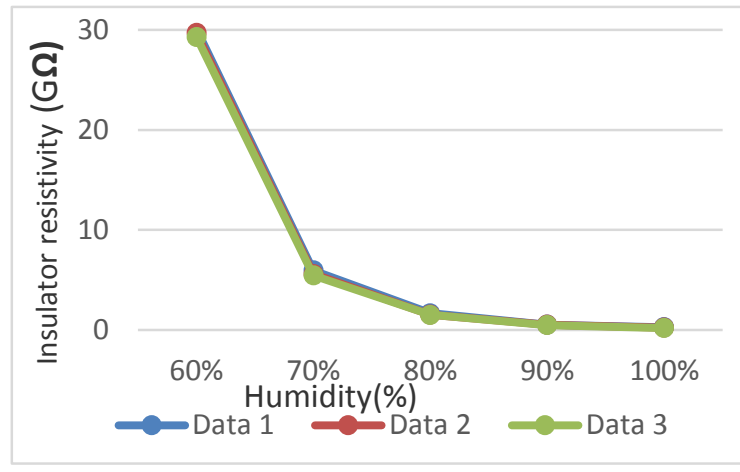

Figure 13. Data from the calculation of insulation resistivity of humidity differences in uniform fine insulators

humidity difference added to the test insulator rises. The value of insulation resistance is inversely proportional to the rise in air humidity, and as the humidity of the applied air increases, the air surrounding the insulator surface is damp, resulting in a conductive electrolyte. This makes it possible for the current to pass on the insulator surface. The higher the conductivity of the insulator, the lower the insulator resistance. It can also be inferred that the relationship between air humidity and insulation resistance is inversely proportional, the greater the humidity of the air applied to the insulator, the lower the insulation resistance.

\section{Conclusion}

The amount of leakage current of the epoxy resin insulator with $\mathrm{SiO} 2$ and alumina fillers increases with the rise in test voltage difference, the increase in temperature variation, and the increase in humidity variation added to the insulator. The association between insulation resistance and temperature is inversely associated. The higher the temperature applied to the insulator, the lower the insulation resistance. The association between insulation resistance and air humidity is inversely proportional. The greater the humidity applied to the insulator, the lower the insulation resistance. The quality of the insulators filled with Alumina and $\mathrm{SiO} 2$ is quite good compared to silicon rubber ( $\mathrm{SiR}$ ) insulators, especially the resistance of leakage currents to temperature variations, such that the insulator can be used and reproduce.

\section{References}

Badachi, Chandrashekhar., Dixit, Pradipkumar., (2016). Prediction of Pollution Flashover Voltages of Ceramic String Insulators Under 


\section{TEKNIK, 42 (1), 2021, 19}

Uniform and Non-Uniform Pollution Conditions. Journal of Electrical Systems and Information Technology 3, 270-281.

Qiao, Xinhan,. Zhang, Zhijin,. Jiang, Xingliang,. Sundararajan, Raji,. You, Jinwei., (2020). DC Pollution Flashover Performance of HVDC Composite Insulator Under Different NonUniform Pollution Conditions. Electric Power Systems Research 185, 106351SSN 0378-7796.

Syakur. A., Hermawan, Sutanto (2017). Study on Tracking Time of Epoxy Resin Insulating Material Under Artificial Accelerated Aging. ICHVEPS 2017; International Conference on High Voltage Engineering and Power Systems. (pp.503-507). Bali, Indonesia.

Gao. Y., Wang. X., Li. N., Xu. B., Wang. J., Du. B (2019). Characterization Method for Carrier Trap and the Effect on Insulation Breakdown Within Polymer Insulating Materials: A Review. Gaodianya Jishu/High Voltage Engineering Volume 45. Issue 7. 31 July 2019. Pages 2219-2230.

Taryo, Syakur. A., Hermawan (2018). Comparison Study on Leakage Current of $20 \mathrm{kV}$ Silicon Rubber and Epoxy Resin Insulator under Dry and Wet Condition. 5th International Conference on Information Technology. Computer and Electrical Engineering. ICITACEE 2018. 13 December 2018, Article number 8576976, Pages 260-263..

Utami, PN. (2018). Analisis Arus Bocor Isolator Silicon Rubber Dan Resin Epoksi Sistem Distribusi $20 \mathrm{kV}$ Variasi Kelembaban dan Temperatur. Master Thesis. Diponegoro University.

International Electrotechnical Commission (IEC) 61109. Insulators for overhead lines Composite suspension and tension insulators for a.c. systems with a nominal voltage greater than $1000 \mathrm{~V}$ - Definitions, test methods and acceptance criteria. International Electrotechnical Commission. Geneva, 2008 (p. 21).

International Electrotechnical Commission (IEC) 60335-1. Household and similar electrical appliances - Safety - Part 1: General requirements. International Electrotechnical Commission. Geneva, 2020 (p. 13).

International Electrotechnical Commission (IEC) 60950-1. Information technology equipment Safety - Part 1: General requirements. International Electrotechnical Commission. Geneva, 2005 (p. 17).

Arismunandar. A. (2009). Teknik Tegangan Tinggi (p. 91). Jakarta: Pradnya Paramita.

Tobing. Bonggas L. (2012). Peralatan Tegangan Tinggi (Pp. 147-149). Jakarta: PT Gramedia Pustaka Utama.

Suwarno. (2016). Partial Discharge in High Voltage Insulating Materials. International Journal on Electrical Engineering and Informatics Volume 8. Number 1. March 2016 (Pages 147163).

Ananth. A., Ravindran. M. (2016). Investigation of Leakage Current of Insulator Using Artificial Neural Network. International Journal of Engineering Sciences \& Research Technology. August. 2016. Pages 667-672.

Zhijin Zhang, Xinhan Qiao, Yi Zhang, Liang Tian, Dongdong Zhang, Xingliang Jiang (2018). AC flashover performance of different shed configurations of composite insulators under fan-shaped non-uniform pollution. The Institution of engineering and technology (IET) Journals. Volume 3, Issue 3, (Pp. 199 -206). 